\title{
Turbulence in high latitude molecular clouds
}

\author{
S. N. Shore ${ }^{1,2}$, T. N. LaRosa ${ }^{3}$, L. Magnani ${ }^{4}$, \\ R. J. Chastain ${ }^{4}$ and F. Costagliola ${ }^{1}$ \\ ${ }^{1}$ Dipartimento di Fisica "Enrico Fermi", Università di Pisa, Pisa 56127, Italy \\ email: shore@df.unipi.it,francesco.costagliola@df.unipi.it \\ ${ }^{2}$ INFN-Sezione di Pisa \\ ${ }^{3}$ Department of Biological and Physical Sciences, Kennesaw State University, Kennesaw, GA \\ USA \\ ted@avatar.kennesaw.edu \\ ${ }^{4}$ Departiment of Physics, University of Georgia, Athens, GA USA \\ email: loris@physast.uga.edu
}

\begin{abstract}
We summarize a continuing investigation of turbulence in high-latitude translucent molecular clouds. These low mass $\left(\sim 50-100 \mathrm{M}_{\odot}\right)$, nearby $(\sim 100 \mathrm{pc})$, non-star forming clouds appear to be condensing out of the atomic cirrus. Unlike star-forming clouds the velocity fields in the clouds must be driven by external processes. Our detailed mapping of the clouds MBM 3,16 and 40 indicates that the dynamics in these clouds result from the combination of shear-flow and thermal instabilities, not shocks. These clouds also show coherent structures, non-Gaussian PDFs but no clear velocity-size relation. Lastly, the energetics of these clouds indicate that radiative loss may terminate the cascade before local heating takes place.
\end{abstract}

Keywords. turbulence, hydrodynamics, instabilities, ISM: kinematics and dynamics, ISM: clouds, radio lines: ISM

\section{Introduction}

The general problem of interstellar turbulence has changed its character in recent years from a debate over its existence to a wide variety of studies addressing its diagnosis and role in the dynamics of the large scale medium and the regulation of star formation (e.g. Elmegreen \& Scalo 2004, Mac Low \& Klessen 2004). For this symposium, the main reason we should care about the turbulence is its feedback into the regulation process for star formation. When the turbulent cascade is in equilibrium, the effect of the turbulent pressure can far outweigh that of the gas in providing dynamical support for the rest of the cloud.

The signatures of turbulence are usually based on spectral line diagnostics and morphology, often using analytic techniques derived from laboratory experience. In many environments, however, experiments cannot serve as an effective guide because of the role played by strong interactions among outflows from recently formed embedded stars, ionization regions, magnetic fields, and self-gravity. These dominate the dynamics of massive, dark clouds: distributed internal sources can easily power the turbulent motions and wipe out the coherence signatures. These flows are too complicated, and previous studies (e.g. Ori, Miesch \& Bally 1994) find no correlation scale, evidently because of the long range fluid interactions once jets and winds appear. But collectively the translucent clouds present a very different case study. These are dull, boring, clouds - not forming stars yet they show all the usual signatures of large scale internal turbulent motions. As we will discuss, even in the absence of self-gravity, the kinematics show an essential element of a 
turbulent flow, intermittency through the observation of non-Gaussian PDFs, coherent structures, and locally sub-dispersed regions. Finally, we hope the reader will bear in mind that whatever the final mechanism for initiating star formation, the first turbulent dissipative structures to condense in the baryonic component within dynamically stirred dark halos should have looked like translucent clouds before star formation started (e.g. the first stars).

The translucent clouds (hereafter TCs) show ordering and structure on many length scales, hence also involving a variety of different scales of mass and time. On the largest size, up to about $10 \mathrm{pc}$, they are connected with $\mathrm{H}$ I clouds that are often parts of larger complexes. All three of the clouds we have studied, MBM 3, 16, and 40 are parts of much larger filamentary and/or sheetlike structures seen with IRAS $100 \mu$ images. In these clouds we find quite substantial, large scale systematic velocity gradients, $\sim(1-3) \mathrm{km}$ $\mathrm{s}^{-1} \mathrm{pc}^{-1}$, extending to scales of several pc. For MBM 3, at least, there are several sheets that appear in the channel maps at the same velocity with spacings of about $0.5 \mathrm{pc}$ and also smaller scale $(0.1$ to $0.5 \mathrm{pc})$ shear flows that also display sizable velocity gradients but more jetlike (see the movie accompanying Shore et al. 2006). On the next scale down, $\approx 1 \mathrm{pc}$, these clouds display coherent structures and fine structure in velocity and space on typical scale 0.1-0.5 pc. By this we mean regions that are spatially distinct - local column density enhancements that also appear to correspond to volume density peaks for which the internal centroid velocities are subdispersed relative to the line widths for individual profiles. This is especially true for MBM 16 for which several regions show extremely narrow profiles in both ${ }^{12} \mathrm{CO}$ and $\mathrm{H}_{2} \mathrm{CO}$. Finally, these structures show finer scale in the profiles of dense gas tracers such as CS (2-1), CS (1-0), and $\mathrm{HCO}^{+}$. A puzzling feature is the presence of "hairpin structure", commonly observed in terrestrial shear flows (Sreenivasan \& Antonia 1997), for the molecular gas within the H I envelope. This may be a red herring, but it is surprisingly frequent (e.g. MBM 16, LaRosa et al. 1999; MBM 40, Shore et al. 2003, see also Chol Minh et al. 2003; Polaris Flare, Falgarone et al. 1998) and also appears in numerical simulations (such as those reported at this meeting by Vazquez-Semadeni). In Figs. 1 and 2 we show an example of one such atomic structure, the H I cloud in which MBM 40 is embedded, for which an Arecibo map shows correspondence between the CO, the FIR (which agrees well with the CO, Shore et al. 1999) and atomic gas. The $21 \mathrm{~cm}$ emission is far more extended than that specifically associated with the velocity range of the $\mathrm{CO}$ and the molecular gas is concentrated in a 'hole' in the $\mathrm{H}$ I distribution.

\section{Analysis methods: probability distribution functions (PDF) and velocity autocorrelation functions (ACF)}

The velocity probability distribution function (PDF) is a measure of the frequency distribution of velocities, in effect a histogram of the fluctuations. In laboratory (shear) flows the velocity difference PDFs systematically show non-Gaussian behavior (Minier \& Peirano 2001) and in the case of the ISM are a more robust measure of the turbulence (see Miesch, Scalo \& Bally 1998 hereafter MSB99; Falgarone \& Phillips 1990). Regardless of the parent distribution for the velocity fluctuations, their PDFs should approach a Gaussian at sufficiently large lag, when the turbulent motions are completely uncorrelated. In astrophysical studies to date several different schemes have been used to compute this diagnostic, correcting for systematic flows by applying different detrending algorithms to the data. For example, Kitamura et al. (1993), Miesch \& Bally (1994), Miesch et al. (1999), LaRosa, Shore, \& Magnani (1999), and Shore et al. (2003) removed any large scale trends in their maps by computing a mean fitted, or smoothed map, subtracting this 


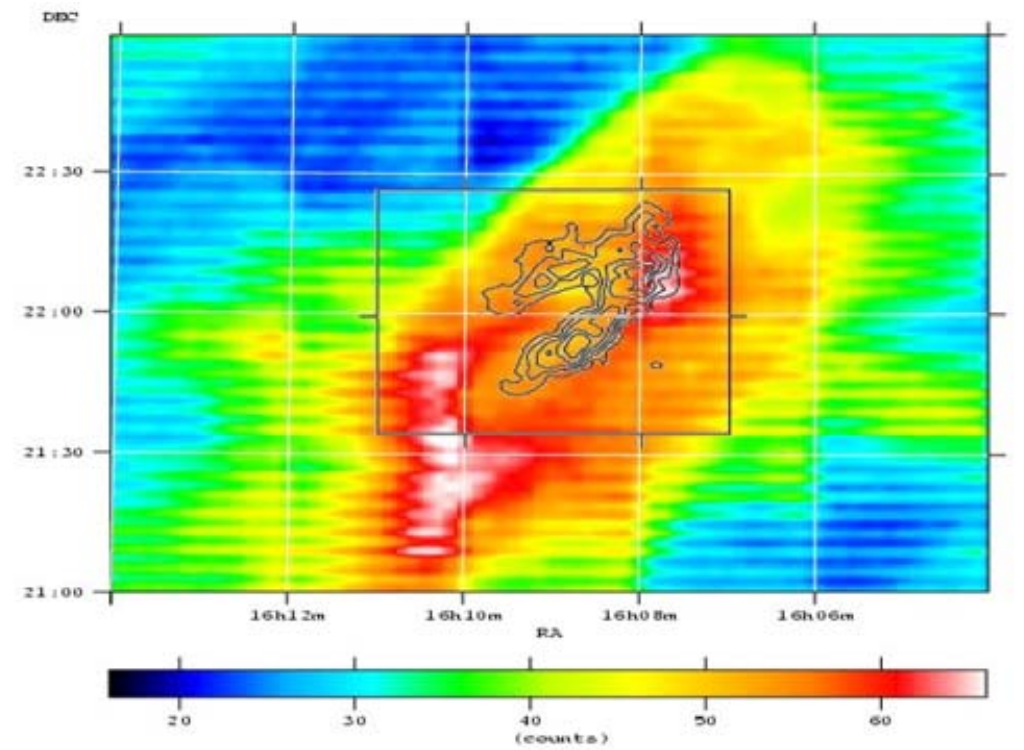

Figure 1. The molecular and atomic relationship for MBM 40. Color represents H I (Arecibo) and contours superposed on the $\mathrm{H}$ I map are $\mathrm{CO}(1-0)$ emission (FCRAO). The H I spans $2.6-3.5 \mathrm{~km} \mathrm{~s}^{-1}$, the velocity range covered by the CO. See Shore et al. (2003) for details.

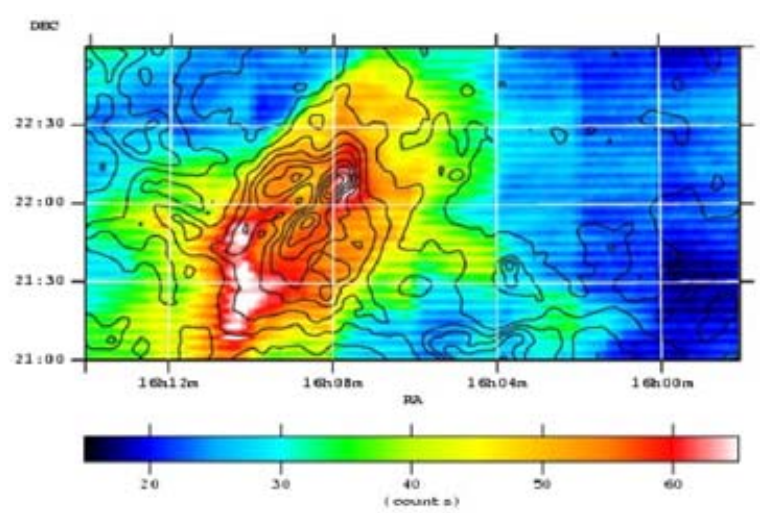

Figure 2. The large-scale environment of MBM 40: H I (Arecibo) and 100 $\mu$ IRAS emission. Same as Figure 1, except that the contours represent IRAS $100 \mu$ emission. The infrared emission is tightly correlated with the total hydrogen column density (atomic plus molecular).

from the original data and analyzing the residual fluctuations. In laboratory turbulence studies this corresponds to the usual Reynolds decomposition that focuses on the fluctuations about the mean flow. Detrending is especially important for correlation analyses since large scale gradients will dominate any correlation and mask correlations generated by turbulence. Alternatively, Ossenkopf \& Mac Low (2002) and Pety \& Falgarone (2003) did not explicitly remove the large scale trends, arguing that on the scale of their maps such flows are themselves part of the overall cascade and should not be separated. If the shear is powering the cascade, however, it is necessary to remove it to see the smallest scales of the correlation in an ACF but not in a PDF analysis (see Minier \& Peirano 2001). 
What do PDFs show that the ACF and Principal Component Analysis (PCA) methods don't? Regardless of the parent distribution for the velocity fluctuations, their PDFs should approach a Gaussian at sufficiently large lag, when the turbulent motions are completely uncorrelated as required by the Central Limit Theorem. This convergence is also a measure of the correlation length independent of an explicit measurement of the correlation function. The PDF can be described by generalized Lévy processes. Lévy processes are characterized by statistical distributions that have no well defined mean or variance. That is they have long tails indicating that large events can occur with appreciable probability. Although a Gaussian PDF can result from a merely chaotic processes, a Lèvy process cannot. The smallest scales sampled by the shift PDFs (Lagrangian) for only a few lags, or from those obtained using smoothed centroid map differences (Eulerian) must be detecting the comparatively small regions in which the cascade terminates and the kinetic energy dissipates.

\section{The velocity-size relation in the translucent clouds}

First suggested in the 1980s, for a variety of reasons one would expect some sort of dynamical connection between the size and velocity dispersion for a virialized and/or turbulent structure. One was found very early in the work on cloud structures, the first proposal by Larson (1981), for a heterogeneous sample, was $\Delta V \sim L^{0.38}$. As Larson noted, this is suggestive of a turbulent cascade $\epsilon=$ constant with $\Delta V \sim L^{1 / 3}$ (RichardsonKolmogorov scaling). Being astrophysicists, it sufficed at first that 0.38 is close enough to $1 / 3$ to be a reasonable explanation. A similar relation results simply from assuming the clouds are virialized regardless of internal activity or scale. But it's not obvious we are seeing a single process, or even a stationary one, in any line of sight and many different environments were combined in this analysis.

Further refinements have changed this simple picture. The signature differs depending on environment. On the largest scale, tens of pc $\alpha=0.5 \pm 0.05$ (Dame et al. 1986, Solomon et al. 1987). Multi-cloud, single tracer: $\alpha=0.31 \pm 0.07$ (Kawamura et al. 1998); $0.26 \pm 0.09$ (Yonekura et al. 1997); $\alpha=0.62 \pm 0.09$ for $3 \leqslant L \leqslant 30$ pc, PCA using FCRAO cubes (Heyer \& Brunt 2004). On the smaller scales, the picture is far less clear. High density cores, $\alpha=0.23 \pm 0.03$ (high mass), $0.53 \pm 0.07$ (low mass) (Caselli \& Myers 1995). In contrast, for the high latitude TCs we don't seem to see anything systematic. For all three clouds, especially our most recent study of MBM 3, the line-widths are independent of the region size! Although this result contrasts sharply with those from the FCRAO survey, presented in this meeting by Heyer, the two sets of results may yet be reconcilable. Why should we see this relation on large and not small scales? On the scale of $>1 \mathrm{pc}$, mixtures of random and systematic motions along any line of sight contribute to the line widths. Systematic motions and gradients are detected on a variety of size scales from velocity centroid maps - when large enough (relative to the intrinsic velocity dispersion) they dominate the line widths. Increasing the surveyed area increases the chance of encountering large gradients, hence $\Delta V$ must increase. In contrast, for the smaller scales, $\Delta V$ is dominated by smaller scale random or turbulent motions, since it is unlikely that small clouds will have more than a single velocity gradient. The velocity dispersion should no longer depend on the size of the sample or its location in the cloud. However, velocity shears and gradients are often unstable, transforming ordered into turbulent motions, so if a cascade develops, any large scale gradients are smeared out, leaving only small scale centroid fluctuations. 


\section{Energetic considerations for these clouds}

The bottom line from these studies is that the driving for the high latitude clouds appears to be external, by large scale shear flows in the H I medium from which these objects are condensing. The scale for the systematic motions, of order parsecs, may also connect several structures within the same larger HI concentration, two questions naturally arise. Is there evidence for any connection between TCs within the same IRAS or HI structure - e.g. shells and/or filaments - and how do these form as cold molecular clouds embedded within the atomic gas?

Where does turbulent dissipation happen? In the original Kolmogorov picture (also called K41), the cascade proceeds from the injection scale to smallest scales at which some form of smooth distribution of fluctuations mimics viscosity and dissipates the energy into localized heating. Between these extremes, the assumption is a monotonic energy transfer between scales, ending in the intermittent regime above the viscous (Kolmogorov) scale (the so-called K62 "second hypothesis"). We can estimate the heating within the cascade by assuming within the inertial subrange transfer rate is given by $\epsilon_{\text {trans }} \sim \rho\left(\sigma_{v, \ell}\right)^{3} \ell^{-1}$ for a velocity dispersion $\sigma_{v, \ell}$ at the associated length $\ell(\leqslant 0.1 \mathrm{pc})$ in the cascade).

Any line profile samples the entire line of sight within a beam (select any $\ell$ ); e.g. $\operatorname{MBM} 3, \sigma_{v} \approx 1.7 \mathrm{~km} \mathrm{~s}^{-1}$, independent of the location so for $n_{H_{2}} \sim 10^{3} \mathrm{~cm}^{-3}, \epsilon_{\mathrm{trans}} \approx$ $3 \times 10^{-23} \mathrm{erg} \mathrm{s}^{-1} \mathrm{~cm}^{-3}$. Assume instead $\sigma_{v} \approx$ (FWHM 1-lag shift PDF, $\ell \approx 0.03 \mathrm{pc}$ ); thus, for MBM 3, $\sigma_{v} \approx 0.4 \mathrm{~km} \mathrm{~s}^{-1}, \epsilon_{\mathrm{trans}} \approx 3 \times 10^{-24} \mathrm{erg} \mathrm{s}^{-1} \mathrm{~cm}^{-3}$. To estimate the rate of energy injection from the shear flow approximate the turbulent viscosity as $\eta_{T}=\rho \ell \sigma_{v}$ taking $\sigma_{v}$ from the line profiles and estimating $\ell$ with the correlation length. Since $\epsilon_{\text {inj }}=$ $\eta_{T}(\Delta V / L)^{2}$ for a velocity gradient $\Delta V / L$ (for these clouds it is typically $\approx 3 \mathrm{kms}^{-1}$ $\left.\mathrm{pc}^{-1}\right), \epsilon_{\mathrm{inj}} \approx 10^{-24} \mathrm{erg} \mathrm{s}^{-1} \mathrm{~cm}^{-3}$. The magnitude of this injection rate seems similar in at least the clouds observed to date.

Numerical simulations of driven MHD turbulence give, for $T \approx 10 \mathrm{~K}$ and the typical observed column density, $7 \times 10^{20} \mathrm{~cm}^{-2}$ (e.g. MBM 3), a CO cooling rate of $10^{-24} \mathrm{ergs}$ $\mathrm{s}^{-1} \mathrm{~cm}^{-3}$ (Juvela, Padoan \& Nordlund 2001). Therefore, CO cooling may keep up with the turbulent energy production. It thus seems likely that at least in some of these clouds the regions of intense dissipation found in some fine structure surveys may be indicating several channels for the transfer of energy, both by loss and by powering chemistry (i.e. Falgarone, these proceedings). It is not clear that in a medium with radiative loss that the cascade must necessarily proceed to small scales and result in heating.

\section{Conclusions}

We end with a summary of our conclusions for turbulence in non-star forming, nonself gravitating translucent clouds. MBM 3, MBM 16, and MBM 40 have been mapped with high spatial $(0.03 \mathrm{pc})$ and velocity resolution $(\leqslant 0.08 \mathrm{~km} / \mathrm{s})$ in ${ }^{12} \mathrm{CO}(1-0){ }^{13} \mathrm{CO}$ (1-0) (NRAO, FCRAO). All show evidence for large-shear flows that we propose powers the turbulent motion. The densest gas is structured into filaments and knots, having a characteristic size of order 0.5 pc. This is the same scale on which we find correlation and also typical of the sub-dispersed regions. Their similarity to laboratory coherent structures may provide another signature of a shear flow origin. The centroid velocity probability distribution function (PDF) is a more precise measure of turbulence. The PDFs exhibit broad wings, consistent with a Lorentzian distribution and showing evidence of non-Gaussian correlated processes. This is a clear signature of intermittency. The density field is more likely the result of thermal instability. The line profiles do not change in going across a filament and there are no centroid changes at the boundaries and, 
evidently, no shocks. And finally, no systematic $\Delta V$-L relation; shears and fine structure can account for the diverse results.

Ultimately the implication for these results for star formation and turbulence is that an external driving, as well as internal stirring, can produce a substantial cascade that dynamically structures molecular clouds. Cosmologically this may be important since the first stars formed out of gas that had been dynamically forced by large scale flows that have nothing to do with stellar input.

\section{Acknowledgements}

We thank the organizing committee for their kind invitation. SNS thanks the IAU for a travel grant and MIUR/Italy and INFN for additional support of the research presented here. SNS and FC thank the staff of OSO and RadioNet for support. TNL thanks the Kennesaw State University Foundation for a travel grant. We especially want to thank Enrique Vazquez-Semadeni, Bruce Elmegreen, John Black, and Edith Falgarone for discussions.

\section{References}

Caselli, P. \& Myers, P. C. 1995, ApJ 446, 665

Chol Minh, Y. C. Y., Kim, H-G, Lee, Y., Park, H., Kim, K-T \& Kim, S. J. 2003, New Astr. 8, 795

Dame, T. M., Elmegreen, B. G., Cohen, R. S. \& Thaddeus, P. 1986, ApJ 305, 892

Elmegreen, B. G. \& Scalo, J. 2004, ARAESA 42, 211

Falgarone, E., Panis, J. F., Heithausen, A. \& Perault, M., et al. 1998, A\&\&A 331, 669

Falgarone, E. \& Phillips, T. G. 1990, ApJ 359, 344

Goodman. A. A., Barranco, J. A., Wilner, D. P. \& Heyer, M. H. 1998, ApJ 504, 223

Heyer, M. H. \& Brunt, C. M., 2004, ApJ 615, L45

Juvela, M., Padoan, P. \& Nordlund, Å. 2001, ApJ 563, 853

Kawamura, A., Onishi, T., Yonekura, Y., Dobashi, K., Mizuno, A., Ogawa, H. \& Fukui, Y. 1998, ApJS 117, 387

LaRosa, T. N., Shore, S. N. \& Magnani, L. 1999, ApJ 512, 761

Larson, R. B. 1981, MNRAS 194, 809

Magnani, L., LaRosa, T. N. \& Shore, S. N. 1993, ApJ 402, 226

McComb, W. D. 1992, The Physics of Fluid Turbulence, (London: Oxford Univ. Press)

Miesch, M. S. \& Bally, J. 1994, ApJ 429, 625

Miesch, M. S., Scalo, J. \& Bally, J. 1999, ApJ 524, 895

Minier, J. \& Peirano, E. 2001, Phys. Rep. 352, 1

Ossenkopf, V. \& Mac Low, M.-M. 2002, A\&A 390, 307

Pety, J. \& Falgarone, E. 2003, A\& $A$ 412, 417

Robinson, S. K. 1991, Ann. Rev. Fluid Mech. 23, 601

Shore, S. N., Magnani, L., LaRosa, T. N. \& McCarthy, M. N. 2003, ApJ 593, 413

Shore, S. N., LaRosa, T. N., Chastain, R. J. \& Magnani, L. 2006, A\&SA 457, 197

Solomon, P. M., Rivolo, A. R., Barret, J. \& Yahil, A. 1987, ApJ 319, 730

Sreenivasan, K. R. \& Antonia, R. A. 1997, Ann. Rev. Fluid Mech. 29, 435

Yonekura, Y., Dobashi, K., Mizuno, A., Ogawa, H. \& Fukui, Y. 1997, ApJS 110, 21

\section{Discussion}

MARov: 1. You have found evidence for dispersion velocities but in your data analysis you use Richardson-Kolmogorov approach applicable for isotropic turbulence only. How do you justify this? Or is it just valid along the line of sight? 2. You invoke shear flow to maintain large eddies formation. What do you think about an other mechanism involving enstrophy acting in the background direction of the cascade energy processes background? 
SHORE: Thanks - lovely questions. 1. The approach is because at the dissipation scale, and near the intermittency scale, it should be far more isotropic than at the source scale. 2. To address these flows are fully $3 \mathrm{D}$ and the underlying topology of the vorticity is poorly known. I agree it will be interesting to study the enstrophy evolution but I suspect it will be observationally very hard. 